\title{
Finding Potential Seeds through Rank Aggregation of Web Searches
}

\author{
Rajendra Prasath^ and Pinar Öztürk \\ Department of Computer and Information Science (IDI) \\ Norwegian University of Science and Technology (NTNU) \\ Sem Sælands Vei 7-9, NO - 7491, Trondheim, Norway \\ \{rajendra, pinar\}aidi.ntnu.no
}

\begin{abstract}
This paper presents a potential seed selection algorithm for web crawlers using a gain - share scoring approach. Initially we consider a set of arbitrarily chosen tourism queries. Each query is given to the selected $N$ commercial Search Engines (SEs); top $m$ search results for each SE are obtained, and each of these $m$ results is manually evaluated and assigned a relevance score. For each of $m$ results, a gain - share score is computed using their hyperlinks structure across $N$ ranked lists. Gain score of each link present in each of $m$ results and a portion of the gain score is propagated to the share score of each of $m$ results. This updated share scores of each of $m$ results determine the potential set of seed URLs for web crawling. Experimental results on tourism related web data illustrate the effectiveness of the proposed seed selection algorithm.
\end{abstract}

Keywords: Web Crawlers, Seed Selection, Link Data, Relevant Judgment.

\section{Introduction}

Online web documents, specifically in textual form, are massively growing and popular search engines process (SEs) these documents using the Information Retrieval (IR) components namely: crawler, indexer and searcher as shown in Figure 1. For a given set of Uniform Resource Locators(URL), the crawler fetches the content of the URLs, the indexer facilitates a comprehensive storage of the extracted content from each URL, through indexes, and the searcher estimates similarity between a user query and the indexed documents. Since searching the web upon each query would require processing millions of pages, a searcher makes the search on a smaller subset of documents collected through seed URLs and returns the top $m$ results. The results of a particular search engine may not include the best possible web pages the user is interested in. Correspondingly, if a user queries for certain information, different search engines provide differently ranked top $m$ search results. Two factors mainly determine the quality of search results: similarity judgment and seed URLs. The former is popularly known as "web ranking". The latter should be a good representative of the web in order to retrieve maximum number of relevant documents. However, it is often not, leading to inadequate relevant results.

\footnotetext{
* Currently at: Dept of CSE, Indian Institute of Technology, Kharagpur - 721 302, India; email: rajendra@cse.iitkgp.ernet.in; drrprasath@gmail.com
} 
Rank aggregation can be seen as the problem of computing the "consensus" ranking of the alternatives, given the individual ranking preferences of several judges. Rank aggregation has been applied in the context of searching and retrieval of web content, such as meta-search, aggregating the ranking functions, spam reduction, word association techniques, airline reservation, ranking restaurants based on different criteria (cuisine, driving distance, ambiance, star or dollar rating, etc) and comparison of various search engines [5]. In this paper, we propose a rank-aggregation based seed selection algorithm for discovering a good representative subset of URLs as potential seeds.

The paper is organized as follows: Next section describes the notion of seed URL and a brief overview of the related research. Section 3 describes the proposed method for iteratively discovering seed URLs. Section 4 describes evaluation methodology and experimental results. Finally, section 5 concludes the paper.

\section{Discovering Potential Seeds}

Web crawlers download web pages by exploring the interconnected hyperlink structure of the World Wide Web. This process is known as web crawling or spidering. From these downloaded pages, search engines extract the content and adds to the index for searching. Since web pages are frequently changed, modified, added and / or deleted, it is necessary to perform the index update through periodic visits of the web content. General and specific purpose search engines apply modern search technologies. Still no single search engine has proven to satisfactorily meet the information needs of users due to the poor coverage and / or ranking of web contents [2]5].

To make the crawling more effective, it is essential to select proper seeds from which the crawler can start its discovery of web pages towards more "related" and less "noisy" web content [8[12]. More recently Zheng et al. [13] proposed a seed selection algorithm based on the analysis of the graph structure of the web. This method assumes that every crawled web page has been assigned a value. A higher value indicates higher quality or higher potential to discover new pages. In addition, the value can be negative if the page is undesirable, such as a spam page. It was shown that the addition of seeds representing "similar" web documents would improve the search results [11]. Hyperlink recommendations have also been used to increase the web coverage [6].

To crawl the specific type of web content, special crawlers like focused crawlers are being used[3]. These crawlers mainly focus on pages that are relevant to a predefined set of topics which shape the list of seed URLs. However focused crawlers heavily rely on the topical locality phenomenon in which web pages on a given topic are usually clustered together, with many links that connect one page to another. Once a good page is found, the crawler can analyze its cluster to retrieve pages on the same topic[7]. Bergmark et al. suggested a tunneling technique which allows the crawler to follow a limited number of bad pages in order to reach the good ones [1]. Recently Yahoo! invented a method to compare and choose seeds amongst potential sites[4]. Revisiting the same seed sites on a regular basis may not result in discovering enough new URLs. 


\section{Proposed Approach Using Rank Aggregation}

In this section we present an algorithm that selects the seeds based on the link analysis of search results each obtained for a randomly selected query. Figure 1 shows the basic web crawler with the proposed modifications.

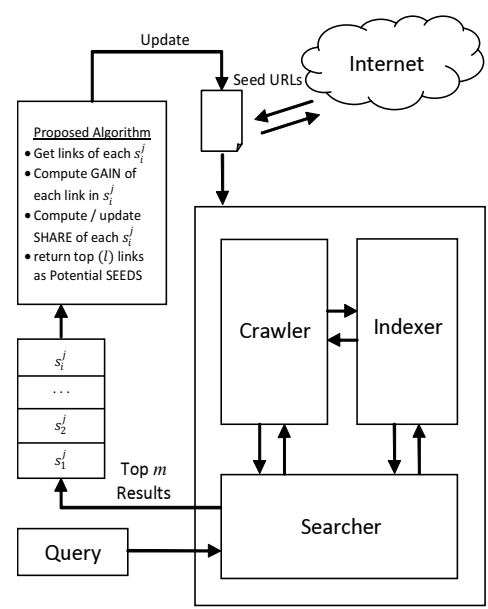

Fig. 1. The proposed algorithm and its role in a search engine
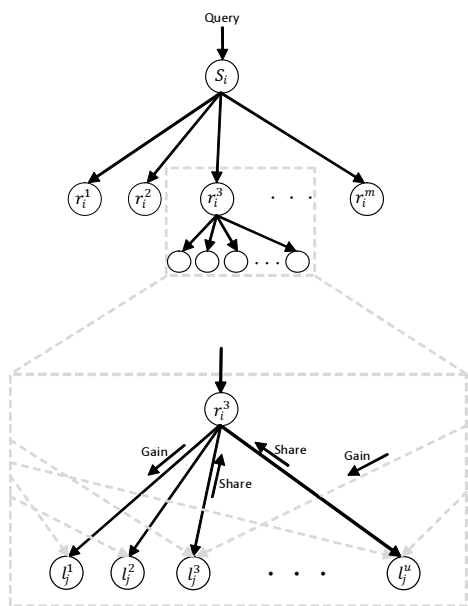

Fig. 2. The proposed gain-share strategy

The algorithm is based on a gain and share mechanism on the links present in the retrieved search results (see Figure 2). The Gain of a link is defined as the score contributed by the number of links pointed to it. Similarly, the Share of a search result is defined as the score that contributes to the seed score of qualified links. Initially, from the arbitrarily selected set of queries $\left\{q_{1}, q_{2}, \cdots, q_{n}\right\}$, each query is issued to $N$ general purpose search engines. For each $q_{p}, 1 \leq p \leq n$, we obtain $N$ ranked sets of URLs: $S=\left\{S_{1}, S_{2}, \cdots, S_{N}\right\}$ where each $S_{i}, 1 \leq i \leq N$ contains a ranked list of top $m$ search results: $S_{i}=\left\{r_{i}^{1}, r_{i}^{2}, \cdots, r_{i}^{m}\right\}$. Each $r_{i}^{j}, 1 \leq j \leq m$, is visited and its relevance as a seed is assigned manually. Then the links, present in each $r_{i}^{j}$, of all $\mathrm{N}$ ranked search results corresponding to the given query $q_{p}, 1 \leq p \leq n$, are collected and gain - share score of each $r_{i}^{j} \in S$ is iteratively computed. For each link $l_{j}^{k}, 1 \leq k \leq u$ ( $u=$ total number of links in $r_{i}^{j}$ ) present in $r_{i}^{j}$, a gain score is computed and a part of this score is propagated to $r_{i}^{j}$ as a share score. This share score updates the seed score of $r_{i}^{j}$ which, in turn, determines the subset of potential URLs for each query. Algorithm 1 presents the details of this mechanism.

It is to be noted that each of the search results, $r_{i}^{j}$, may contain links leading to relevant / related web pages (or may not in the worst case - in which it is a dead end page having no further links to span the web - this would not be a good seed URL and hence discarded directly). Now the crawler is used to get the content of each of the 
retrieved search results. This content is first segmented into blocks and the noisy blocks are eliminated using link - to - text ratio. Here Link - to - text ratio is defined as the ratio between the size of the text tagged with hyperlinks and the text without hyperlinks. This possibly eliminates noisy blocks from the web document using the structural properties of the underlying mark up language and presents the valid blocks. From these valid blocks, outgoing links are collected and used to estimate the gain - share scores.

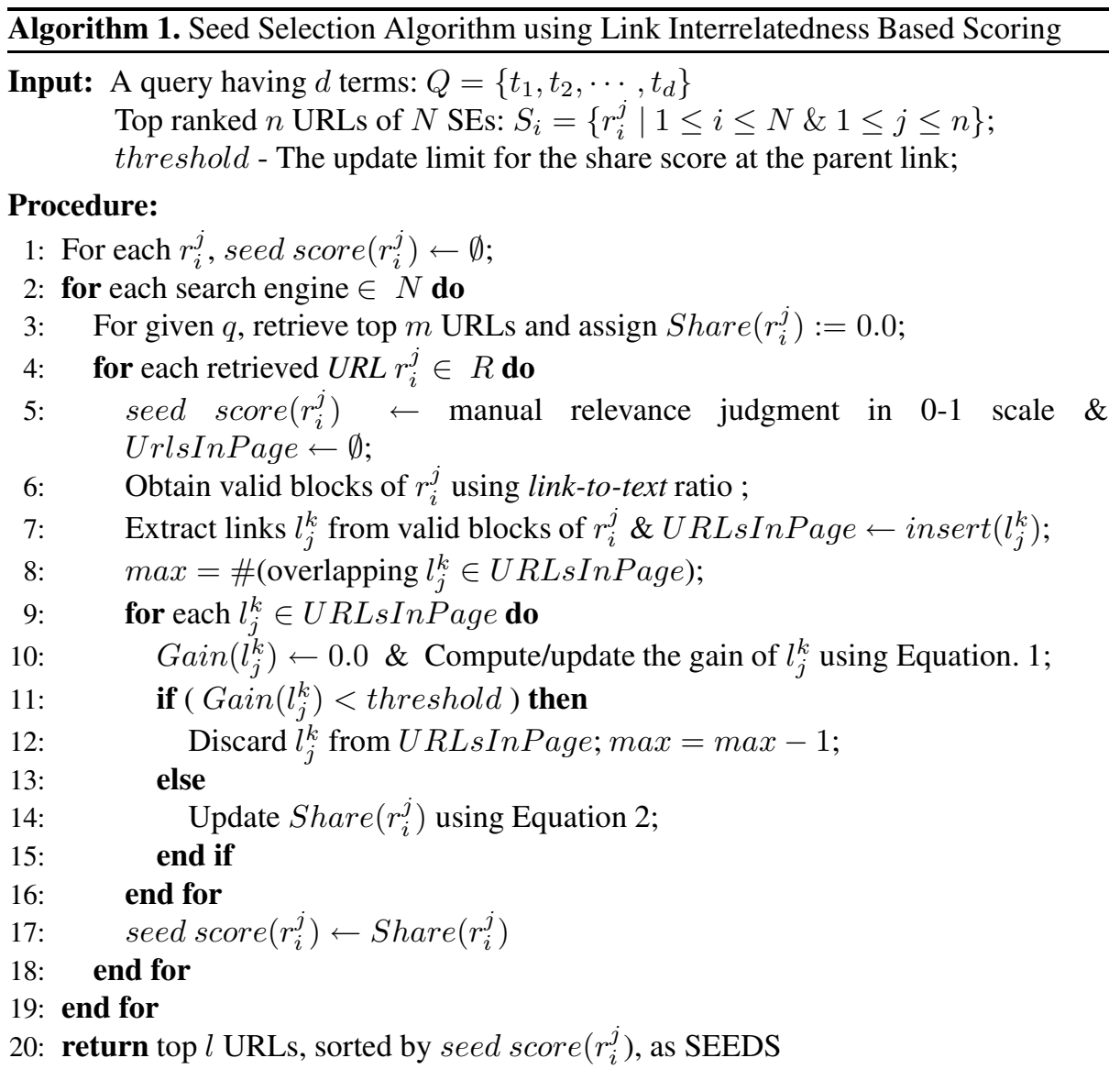

Output: Top $l$ SEED URLs sorted by their seed score in the decreasing order

For each search result, we obtain $m$ URLs (delivered by one of the SEs) each of which is stored in UrlsInPage ${ }_{j}$ where $1 \leq j \leq m$. Then we apply the rank aggregation process on each $l_{j}^{k}$, where $1 \leq k \leq n$ in each search result and obtain the gain scores.

$$
\operatorname{Gain}\left(l_{j}^{k}\right)=\operatorname{Gain}\left(l_{j}^{k}\right)+\frac{\# O L\left(r_{i}^{j}\right)}{\operatorname{rank}\left(r_{i}^{j}\right)} \times \operatorname{seed} \operatorname{score}\left(r_{i}^{j}\right)
$$


where $r_{i}^{j}=P\left(l_{j}^{k}\right)$ - the parent of link $l_{j}^{k}$; \#OL $\left(r_{i}^{j}\right)=$ Total number of outgoing links from $r_{i}^{j} ; \operatorname{rank}\left(r_{i}^{j}\right)=\{v\} \forall v, 1 \leq v \leq n$. Initially, gain is assumed to be zero.

In the proposed algorithm, we have just used the outgoing links from the search engine results $r_{i}^{j}$, but in principle, one could use several levels of outgoing links from $r_{i}^{j}$. Thus the algorithm could be generalized where gain - share score is computed iteratively over a chain of links. Each of these links propagate a certain amount of share of their gain score to its parent (actual search results) to support it as a good seed. We term the final share score as the seed score.

$$
\operatorname{Share}\left(r_{i}^{j}\right)=\operatorname{Share}\left(r_{i}^{j}\right)+\left(\operatorname{Gain}\left(l_{j}^{k}\right) / \max \right)
$$

where $\max$ - the number of overlapping outgoing links of search results across $N$ search result sets for each corresponding query.

This score will be shared with the corresponding search result in the bottom up fashion and will support the search result for becoming a good candidate in seed selection. This is repeated for all links present in all subsets and then the top $m$ search results of $N$ search engines. Once we complete the gain - share computations for all top $m$ search results which are then aggregated and based on their new seed score and top $m$ links were selected as the potentially good seed URLs.

\section{Evaluation and Discussions}

We have selected 10 tourism queries (in English) from Cross Lingual Information Access (CLIA) Project - a major project for CLIA in Indian languages, funded by the Government of India, and being executed by a consortium of several academic institutions and industrial partners 1. Each query is presented in three forms: title the actual query, desc - the expanded query and narr - the narration of the query. Here we consider each query either with ( title and / or desc). The structure of each query is shown in Figure 3 and selected list of queries are given in the Figure.4

\subsection{Dataset and Evaluation Strategy}

We have collected URLs of top $n=30$ search results for each of the queries given in Figure 4 using four commercial search engines: Google, Yahoo!, Bing and Cuil during September 2010. Thus for each query, we have collected 120 ( $=4$ search engines each with top 30) search results which may be disjoint or may partly overlap across the search results of different search engines. We repeated this process for all 10 queries listed in the Figure 4 and collected 1200 web pages. Some of the links were obsolete, modified or removed. All these 1200 web pages were visited manually and relevance of each was judged in the scale of real numbers between 0 and 1 with 0.1 steps. The actual overlap among the search results of 4 search engines are listed in Table 1 and the rank of these overlapping URLs across 4 search results are different. We measure the goodness of seeds (in turn, the goodness of the crawl created by the ranked seed set) by Precision measure. Precision $(\mathrm{P})$ is the fraction of retrieved documents that are relevant. We measure P@d ("Precision top $d$ documents") with $d=10$.

\footnotetext{
${ }^{1}$ http://www.clia.iitb.ac.in/clia-latest
} 


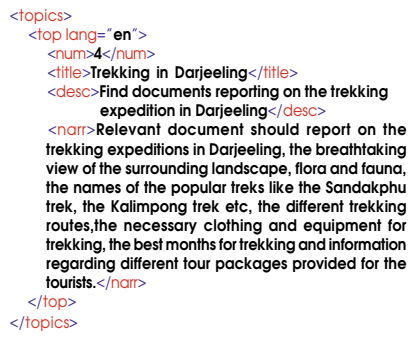

Fig. 3. Query in XML format

\begin{tabular}{|c|l|}
\hline QID & Query (title) \\
\hline Q1 & Amritsar and the Golden Temple \\
Q2 & Rishikesh Water Rafting \\
Q3 & Toy train Shimla \\
Q4 & Mumbai Ganesh Festival \\
Q5 & Sunderbans National Park and its tourist \\
& attractions \\
Q6 & Cuisine of Tamil Nadu \\
Q7 & Goa its beautiful beaches \\
Q8 & Sun Temple at Konark \\
Q9 & Meghalaya and its virgin beauty \\
Q10 & Trekking in Darjeeling \\
\hline
\end{tabular}

Fig. 4. Selected 10 CLIA Queries

Table 1. Overlapping URLs among 300 [10 Queries $\times$ Top 30] Results

\begin{tabular}{|c|c|c|c|c|}
\hline \# Engines & Google & Yahoo! & Bing & Cuil \\
\hline Google & 0 & 90 & 108 & 38 \\
\hline Yahoo! & 90 & 0 & 108 & 29 \\
\hline Bing & 108 & 108 & 0 & 32 \\
\hline Cuil & 38 & 29 & 32 & 0 \\
\hline
\end{tabular}

\subsection{Relevance Judgement}

During the manual relevant judgement, we focus particularly on the search results leading to the "intent" of user's queries. Since the domain being tourism, it is natural to get lots of web content related to tour packages or planning trips (schedulers), or having forms for booking accommodation or transport. So we have given less importance to such contents and more to the focused content of the web documents. During manual evaluation, we have made some observations which mimic the characteristics of the commercial search engines. Some of the search results for the given query leads to video information and may be visually related to the given query. But it is not useful for the systems handling textual content or users having the intent in exploring the history behind the query. In a query related to "trekking", most of the pages contain schedules and plans about the trekking packages offered at various nearby hilly places. Forum discussions / answers describing the user experiences on a specific feature - may be event or place or hotel - are obtained for queries having proper name.

We eliminated the outdated or invalid results by marking it as -1. A few search results contained information about the subtopic of the given query than fetching the overall intent of the query. Such pages were proportionately marked with score deductions. The search results having site replications at different URLs were ranked in the list. For example, page having URL like "http://www.abc.com" and "http://abc.com" were considered as single entity and it is hardly possible to avoid this in the automated web crawling. 


\subsection{Discussions}

Here we present the experimental results of the proposed method on selected tourism related queries. Out of total 1200 search results [ $=10$ Queries x top 30 results x 4 search engines], we have identified 320 links that are common across search results of different search engines. Hence $26 \%$ of same search results repeat on an average for top 10 queries. We have noticed similar amount of search results between Google and Yahoo! search engines. However the number of matching results between Google and Bing is high and Google and Cuil is low (please refer to Table 1). This forms a fair distribution among the search results across different search engines for different queries. During the preliminary analysis, we found that if more number of child link influence the gain - share score, then it is essential to modify the threshold so that more and more hyperlinked pages, even though they are irrelevant, would hardly dominate the score propagation.

First we selected top 40 urls for each query and crawled them to the depth 2 . Now for each query, we have manually checked the precision @ top d $(=10)$ documents score for selected queries(refer to Figure 6). The proposed method diverges in some cases for example, Q10 - "Trekking in Darjeeling”, there are lots of pages with Nepal expeditions and Himalayan expeditions rather than focusing on Sandakphu and Kalimpong Treks. We have done this experiment with only 1200 relevant judged web documents and hence the diversity of the seed selection would be attempted with more data.

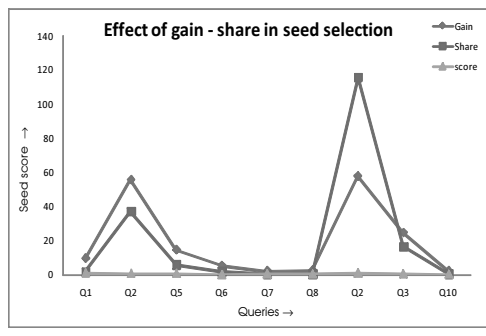

Fig. 5. Seed Score variations for the Selected Queries

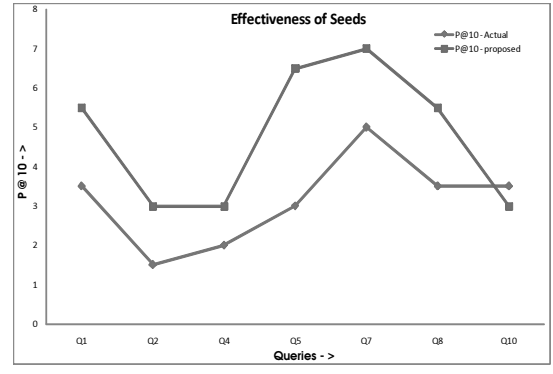

Fig.6.P@10 Scores

Figure 5 shows the preliminary results of our experiments towards seed selection among the selected queries. Here we have highlighted special cases in which manual judgment score is lower, but the gain - share score is considerably higher. This indicates that this seed could be a better candidate for selecting as the seed URL. Similarly the seed for the query $Q 8$ which ranked 7 in the ranked list with partial relevance can be a better choice due to its link structures. These links carry forward to the relevance links in a few steps. In the sequel, we would like to experiment more in comparison with the recent web crawler seed selection algorithms. 


\section{Conclusion}

In this paper, we proposed a seed selection algorithm for web crawlers using link data with gain - share based approach. The proposed method would capture the potential seeds from the ranked search results of commercial search engines in an indirect way. This approach could also be generalized to find better seeds by accounting the crawling to various depths. Analyzing the commercial search results can model the characteristics in finding good candidates towards computing the "interestingness" of a page(in turn, relevance to the user query). In our future work, attempts would be made to dynamically modify, update and / or remove the outdated URLs [9]10].

\section{References}

1. Bergman, M.K.: The deep web: Surfacing hidden value. Journal of Electronic Publishing 7(1) (August 2001)

2. Brin, S., Page, L.: The anatomy of a large-scale hypertextual web search engine. In: WWW7: Proceedings of the seventh international conference on World Wide Web 7, pp. 107-117. Elsevier Science Publishers B.V., Amsterdam (1998)

3. Chakrabarti, S., van den Berg, M., Dom, B.: Focused crawling: a new approach to topicspecific web resource discovery. Comput. Netw. 31(11-16), 1623-1640 (1999)

4. Dmitriev, P.: Host-based seed selection algorithm for web crawlers. US Patent (US20100114858A1) (May 2010)

5. Dwork, C., Kumar, R., Naor, M., Sivakumar, D.: Rank aggregation methods for the web. In: WWW 2001: Proceedings of the 10th international conference on World Wide Web, pp. 613-622. ACM, New York (2001)

6. Hawking, D., Craswell, N.: Which search engine is best at finding online services? In: Proceedings of WWW10, Hong Kong (2001)

7. Micarelli, A., Gasparetti, F.: Adaptive focused crawling. Springer, Heidelberg (2007)

8. Niu, C., Li, W., Ding, J., Srihari, R.K.: A bootstrapping approach to named entity classification using successive learners. In: ACL 2003: Proceedings of the 41st Annual Meeting on Association for Computational Linguistics, pp. 335-342. Association for Computational Linguistics, Morristown, USA (2003)

9. Pal, S.K., Talwar, V., Mitra, P.: Web mining in soft computing framework: relevance, state of the art and future directions. IEEE Transactions on Neural Networks 13(5), 1163-1177 (2002), http://dx.doi.org/10.1109/TNN.2002.1031947

10. Rumelhart, D.E., Hinton, G.E., Williams, R.J.: Learning internal representations by error propagation. MIT Press, Cambridge (1986)

11. Smucker, M.D., Allan, J.: Using similarity links as shortcuts to relevant web pages. In: SIGIR 2007: Proceedings of the 30th annual international ACM SIGIR conference on Research and development in information retrieval, pp. 863-864. ACM, New York (2007)

12. Yangarber, R.: Counter-training in discovery of semantic patterns. In: ACL 2003: Proceedings of the 41st Annual Meeting on Association for Computational Linguistics, pp. 343-350. Association for Computational Linguistics, Morristown, USA (2003)

13. Zheng, S., Dmitriev, P., Giles, C.L.: Graph based crawler seed selection. In: WWW 2009: Proceedings of the 18th international conference on World wide web, pp. 1089-1090. ACM, New York (2009) 